\section{BMJ Open}

Ophthalmology

\title{
Bandage contact lens and topical steroids are risk factors for the development of microbial keratitis after epithelium-off CXL
}

Argyrios Tzamalis, ${ }^{1}$ Vito Romano, ${ }^{1}$ Robert Cheeseman, ${ }^{1}$ Riccardo Vinciguerra, ${ }^{1}$ Mark Batterbury, ${ }^{1,2}$ Colin Willoughby, ${ }^{1,2}$ Timothy Neal, ${ }^{3}$ Sajjad Ahmad, ${ }^{1,2}$ Stephen Kaye $\mathrm{e}^{1,2}$

To cite: Tzamalis A, Romano V, Cheeseman R, et al. Bandage contact lens and topical steroids are risk factors for the development of microbial keratitis after epithelium-off CXL. BMJ Open Ophthalmology 2019;4:e000231. doi:10.1136/ bmjophth-2018-000231

Received 7 October 2018 Revised 15 December 2018 Accepted 10 January 2019
Check for updates

(C) Author(s) (or their employer(s)) 2019. Re-use permitted under CC BY-NC. No commercial re-use. See rights and permissions. Published by BMJ.

'St Paul's Eye Unit, Royal Liverpool University Hospital, Liverpool, UK

${ }^{2}$ Department of Eye and Vision Science, Institute of Ageing and Chronic Disease, University of Liverpool, Liverpool, UK ${ }^{3}$ Department of Medical Microbiology, Royal Liverpool University Hospital, Liverpool, UK

Correspondence to Dr Argyrios Tzamalis; argyriostzamalis@yahoo.com

\section{ABSTRACT}

Objective To investigate the role of bandage contact lenses (BCL) and topical steroids as risk factors for the development of microbial keratitis after epithelium-off corneal collagen cross-linking (CXL).

Methods and Analysis Patients undergoing CXL between February 2011 and July 2017 were included. Patients were divided into two groups: those who were treated postoperatively with a BCL, topical antimicrobial and steroids (group 1) and those who received only a topical antimicrobial until healing of the epithelial defect before introduction of topical steroids (group 2).

Results 1273 eyes of 964 patients were included. Group 1 comprised 316 eyes and group 2 comprised 957 eyes. There were no significant differences in the presence of persisting corneal haze or scarring between the two groups $(p=0.57)$. Microbial keratitis occurred in nine eyes $(0.71 \%$ of eyes) of eight $(0.83 \%)$ patients (one case was bilateral) out of 1273 eyes. Staphylococcus aureus was cultured from corneal scrapes in seven out of nine $(77.8 \%)$ cases and from contiguous sites in the two cases. All cases occurred in group 1 (incidence $=2.85 \%$ ) and none in group 2 ( $p<0.0001)$. A greater proportion of patients who developed microbial keratitis were atopic $(75 \%, p=0.4)$. Conclusion The use of BCL and topical steroids prior to healing of the epithelium is a significant risk factor for microbial keratitis. $S$. aureus is the most common microorganism and is likely to originate from an endogenous site. Not using a BCL and delaying the introduction of topical steroids until epithelial healing significantly reduce the risk of developing microbial keratitis without increasing the risk of persistent corneal haze.

\section{INTRODUCTION}

Introduced by Wollensak et al in 2003, corneal collagen cross-linking (CXL) has become accepted as a useful technique to reduce the risk of progression of keratoconus and other types of corneal ectasia. ${ }^{12}$ Previous reports have demonstrated the efficacy of CXL to stabilise disease progression, to prevent deterioration of vision and to reduce the need for corneal transplantation. ${ }^{3-6}$

\section{Key messages}

What is already known about this subject?

- Epithelial removal in epithelium-off corneal collagen cross-linking (CXL) may predispose to microbial keratitis.

Although the use of bandage contact lens and topical steroids is useful in the postoperative care, reducing pain and inflammation, there is currently insufficient evidence to which extent they may predispose to the incidence of microbial keratitis.

\section{What are the new findings?}

- The results of our study, which is the largest to date series on microbial keratitis following epithelium-off CXL, demonstrate that postoperative treatment protocol plays a substantial role in preventing this possibly sight-threatening complication.

\section{How might these results change the focus of research or clinical practice? \\ - The use of bandage contact lens and topical steroids in the early postoperative period may increase the risk of developing microbial keratitis and should be avoided in terms of safety.}

Although there are several reports of performing CXL with an intact epithelium (transepithelial or epithelium-on), CXL with epithelial removal (epithelium-off) is still considered to be the gold standard of treatment. ${ }^{1267}$ Removal of the epithelium, however, is associated with a significant risk of epithelial healing problems and may increase the risk of corneal infections, as one of the main physical barriers to microbes and other pathogens is removed. ${ }^{89}$ Indeed, the development of microbial keratitis (MK) is one of the most serious complications following CXL. ${ }^{1011}$

Several risk factors for the development of microbial keratitis following CXL have been proposed. ${ }^{8-11}$ These include the presence of 
an epithelial defect, use of a bandage contact lens (BCL), conditions that may prolong the epithelium healing process such as atopic conjunctivitis, diabetes mellitus or the use of anaesthetic eye-drops, and postoperative steroid use. ${ }^{8-11}$ There is also the potential temporary loss of immune cells in the cornea following treatment. In this study, we investigated the incidence of microbial keratitis in patients undergoing epithelium-off CXL for keratoconus with and without postoperative BCL and steroids, and associated micro-organisms.

\section{PATIENTS AND METHODS}

All patients who had undergone CXL for keratoconus from February 2011 to July 2017 at The Royal Liverpool University Hospital, UK were included. The main criterion for patients being offered CXL was an evident progression of keratoconus demonstrated by a change in the curvature within the cone area of at least 1 dioptre (D) on instantaneous map when measured at least 3 months apart. Exclusion criteria for CXL were minimal corneal pachymetry of less than $380 \mu \mathrm{m}$, recent history of corneal infection, concomitant ocular or systemic autoimmune disease, and pregnancy or breast feeding. A corneal infiltrate with an overlying epithelial defect, ocular discharge, anterior chamber inflammatory reaction, conjunctival injection and subjective symptoms were considered as clinically suspected microbial keratitis.

We previously described the CXL protocol used in our centre. Briefly, it involves removal of corneal epithelium with $20 \%$ alcohol followed by $15 \mathrm{~min}$ of $0.1 \%$ riboflavin (VibeX Rapid). ${ }^{12}$ Corneas were irradiated with Ultraviolet A $365 \mathrm{~nm}$ light using Avedro's KXL (Avedro, Massachusetts, USA) machine at an irradiance of $6 \mathrm{~mW} / \mathrm{cm}^{2}$ for 15 min, delivering a total of $5.4 \mathrm{~J} / \mathrm{cm}^{2}$. The CXL procedures were performed in an aseptic room specially designed and used for CXL following strict hygiene and sterilisation rules. All instruments and materials used during each procedure were sterile, and povidone iodine was applied prior to CXL both to the conjunctival fornices $(5 \%)$ as well as to the surrounding skin $(10 \%)$.

Patients were divided into two groups according to the postoperative treatment that they received. Group 1 included cases in which postoperative management consisted of chloramphenicol $0.5 \%$ eye-drops every 2 hours for the first 2 days and then four times a day for 1 week, the use of a BCL (SB18, Menicon) and dexamethasone $0.1 \%$ eye-drops four times a day for 1 month starting from day 1 . Patients were reviewed on days 2 and 7 and the BCL was removed subject to satisfactory epithelial healing. In group 2, patients received chloramphenicol $1 \%$ preservative-free ointment 2 hourly for the first 2 days and then four times a day for 1 week, followed by $0.1 \%$ dexamethasone eye-drops four times daily for 1 month. All patients were reviewed 2 and 7 days after CXL.

In patients who developed a clinically suspected microbial keratitis following CXL, corneal scrapes were collected for smear and culture. Swabs were also taken from the conjunctival fornices, anterior nares and pharyngeal arches for possible endogenous sources of infection. Patients were commenced on $1 \%$ teicoplanin eye-drops and $0.3 \%$ ciprofloxacin ointment hourly for 24 hours and then hourly only during waking hours according to the clinical response and microbiological results. Possible risk factors were recorded in every case and data were enrolled in a multivariate analysis. Confocal microscopy was performed in all cases.

Statistical analysis was performed with MedCalc Statistical Software V.14.8.1 (MedCalc Software bvba, Ostend, Belgium) and SPSS V.19.0 for Windows. Data are given as mean \pm SD. Normality was checked using the Kolmogorov-Smirnov test. When parametric analysis was possible, the Student's t-test was used to compare the outcomes between two independent samples. Categorical variables were compared using the Fisher's exact probability test. Non-parametric Kruskal-Wallis and Mann-Whitney tests were also used to examine the associations between categorical variables and continuous or ordered outcomes. A binary logistic regression model was used to test the proposed risk factors for significance. A $p$ value of $<0.05$ was considered significant.

\section{RESULTS}

The study included 1273 eyes of 964 patients treated with epithelium-off CXL between February 2011 and July 2017: 316 eyes of 215 patients (47\% of bilateral treatment) in group 1 and 957 eyes of 749 patients (27.8\% of bilateral treatment) in group 2. There were no significant differences between the two groups with regard to age, gender, atopic history, maximum simulated keratometric values, minimum corneal thickness and history of microbial keratitis (table 1). Following treatment, there were no significant differences in the presence of persisting corneal haze or scarring between the two groups $(p=0.57)$, when cases of microbial keratitis were excluded. There were no non-infective-related serious adverse events or reactions.

A microbial keratitis occurred in 9 eyes $(0.71 \%$ of eyes) of $8(0.83 \%)$ patients ( 1 case was bilateral) out of 1273 eyes. All the nine cases of microbial keratitis occurred in group 1 (incidence of $2.85 \%$ ) and none in group $2(\mathrm{p}<0.0001$; table 1$)$. Of the eight patients who developed a microbial keratitis, five had undergone bilateral simultaneous CXL and three patients had had unilateral treatment in one eye only at the time of the development of microbial keratitis. The main characteristics of these patients are shown in tables 2 and 3 . There was a 7:1 male preponderance and the average age was 21.5 years (range: $12-28$ years). All were diagnosed between 1 and 5 days (mean 3.75 days) after CXL (table 3). An epithelial defect with surrounding infiltrate was present in all cases (figure 1). Six eyes had central ulcers $(66.7 \%)$, and a hypopyon was present in three eyes $(33.3 \%)$. The only type of micro-organism isolated was Staphylococcus aureus, which was isolated from the cornea in seven cases, from the conjunctiva in two patients, the nose in three patients and the pharynx 
Table 1 Demographics and clinical characteristics data of patients undergoing CXL: comparison between group 1 and group 2 , as well as between total and cases with microbial keratitis (MK)

\begin{tabular}{|c|c|c|c|c|c|c|}
\hline & Group 1 & Group 2 & $\begin{array}{l}\text { P value } \\
\text { (group 1 vs } \\
\text { group 2) }\end{array}$ & Total & $\begin{array}{l}\text { Cases with } \\
\text { keratitis }\end{array}$ & $\begin{array}{l}\text { P value } \\
\text { (keratitis vs } \\
\text { total) }\end{array}$ \\
\hline Patients (n) & 215 & 749 & & 964 & 8 & \\
\hline Eyes (n) & 316 & 957 & & 1273 & 9 & \\
\hline Age (years) & $23.8 \pm 6.5$ & $24.3 \pm 6.6$ & $0.33^{*}$ & $24.3 \pm 6.6$ & $21.5 \pm 6$ & $0.23^{*}$ \\
\hline Gender (male/female) & $138 / 77$ & $553 / 196$ & $0.27 \dagger$ & $691 / 273$ & $7 / 1$ & $0.79 \dagger$ \\
\hline Atopy & $109 / 215(50.7 \%)$ & $354 / 749(47.3 \%)$ & $0.64 \dagger$ & $463 / 964(48 \%)$ & $6 / 8(75 \%)$ & $0.4 \dagger$ \\
\hline Bilateral treatments & $101 / 215(47 \%)$ & $208 / 749(27.8 \%)$ & $0.006 \dagger$ & $309 / 964$ (32.1\%) & $1 / 8(12.5 \%)$ & $0.7 \dagger$ \\
\hline History of MK & $2 / 316$ & $4 / 957$ & $0.64 \dagger$ & $6 / 1273$ & $0 / 9$ & $1.0 \dagger$ \\
\hline Kmax (dioptres) & $51.7 \pm 6.2$ & $51.2 \pm 6.7$ & $0.24^{*}$ & $51.23 \pm 6.4$ & $53.2 \pm 7$ & $0.36^{*}$ \\
\hline Kmean (dioptres) & $49.72 \pm 5.7$ & $49.39 \pm 7.2$ & $0.46^{*}$ & $49.51 \pm 5.5$ & $50.32 \pm 6.3$ & $0.66^{*}$ \\
\hline $\mathrm{CCT}(\mu \mathrm{m})$ & $501.3 \pm 52$ & $502.7 \pm 59$ & $0.71^{*}$ & $502.4 \pm 57$ & $492.7 \pm 53$ & $0.61^{*}$ \\
\hline CTmin $(\mu \mathrm{m})$ & $469.8 \pm 53$ & $473.9 \pm 49$ & $0.21^{*}$ & $473.6 \pm 56$ & $452.5 \pm 29$ & $0.26^{*}$ \\
\hline Corneal haze/scarring & 1/307 (0.3\%)‡ & $2 / 957(0.2 \%)$ & $0.57 \dagger$ & & & \\
\hline Microbial keratitis & 9/316 (2.85\%) & 0/957 (0\%) & $0.000004 \dagger$ & & & \\
\hline
\end{tabular}

*Assessed with Student's t-test and Mann-Whitney test.

†Assessed with Fisher's exact test.

$\ddagger$ Cases with microbial keratitis were excluded in this analysis.

CCT, central corneal thickness; CTmin, minimum corneal thickness; CXL, corneal collagen cross-linking; Kmax, preoperative maximum simulated keratometric value; Kmean, preoperative average simulated keratometric value.

in one patient (table 3). No specific findings were identified on confocal microscopy. The mean ulcer healing time was 5.3 days (range: 2-20 days). The presence of atopic disease was higher, although not statistically significant $(\mathrm{p}=0.4)$, in those patients who developed a microbial keratitis (six out of the eight patients, ie, $75 \%$ ) as compared with those who did not (48\%). The mean change in visual acuity (converting Snellen to logarithm of the minimum angle of resolution [logMAR] estimate) between pre-CXL and their most recent appointment post-CXL was a worsening of $0.3 \log$ MAR (an improvement of $-0.78 \log$ MAR at best and a worsening of 0.48 $\log$ MAR at worst). No case of reduced compliance to the postoperative treatment protocol was noted among patients with keratitis.

Age, gender, preoperative minimum corneal thickness, history of atopic disease, bilaterality of treatment and preoperative maximum simulated keratometric value were not found to be significant risk factors associated with the development of microbial keratitis (table 4). The only variable that yielded statistical significance was the use of BCL and steroids before epithelial healing $(\mathrm{p}=0.005)$.

Table 2 Detailed demographics of individual cases with microbial keratitis following CXL

\begin{tabular}{|llllllllll}
\hline Patients & Gender & Age & Kmax (dpt) & Kmin (dpt) & Kmean (dpt) & CTmin ( $\boldsymbol{\mu m}$ ) & $\begin{array}{l}\text { VA before CXL } \\
\text { (logMAR) }\end{array}$ & $\begin{array}{l}\text { VA last visit } \\
\text { (logMAR) }\end{array}$ & $\begin{array}{l}\text { VA change } \\
\text { (logMAR) }\end{array}$ \\
\hline Patient 1 & Male & 20 & 47.1 & 41.3 & 44.4 & 454 & 0.18 & 0.18 & 0 \\
Patient 2 & Male & 14 & - & - & 54.7 & 487 & 1 & 1.3 & 0.3 \\
\hline Patient 3 & Male & 23 & 43.6 & 41.8 & 43.4 & 476 & -0.08 & -0.18 & -0.1 \\
Patient 4 & Male & 28 & 64.6 & 53.3 & 50.2 & 486 & 0.6 & 0.6 & 0 \\
Patient 5 & Male & 12 & - & - & 64.8 & 412 & 1.78 & 1 & -0.78 \\
Patient 6 & Female & 28 & 49.8 & 44.8 & 47.7 & 463 & 0.3 & 0.18 & -0.12 \\
Patient 6 & Female & 28 & 55.8 & 46.0 & 49.7 & 447 & 0.18 & 0.3 & 0.12 \\
\hline Patient 7 & Male & 21 & 57.0 & 50.9 & 48.5 & 445 & 0.3 & 0.78 & 0.48 \\
\hline Patient 8 & Male & 26 & 54.7 & 46.0 & 49.5 & 403 & 0.1 & 0.48 & 0.38 \\
\hline
\end{tabular}

-, denotes missing or unavailable data; negative VA change denotes an improvement in logMAR acuity from VA before CXL to VA at last visit. CTmin, minimum corneal thickness; CXL, corneal collagen cross-linking; Kmax, preoperative maximum simulated keratometric value; Kmean, preoperative average simulated keratometric value; Kmin, preoperative minimum simulated keratometric value; VA, visual acuity; $\mathrm{dpt}$, diopters; logMAR, logarithm of the minimum angle of resolution. 


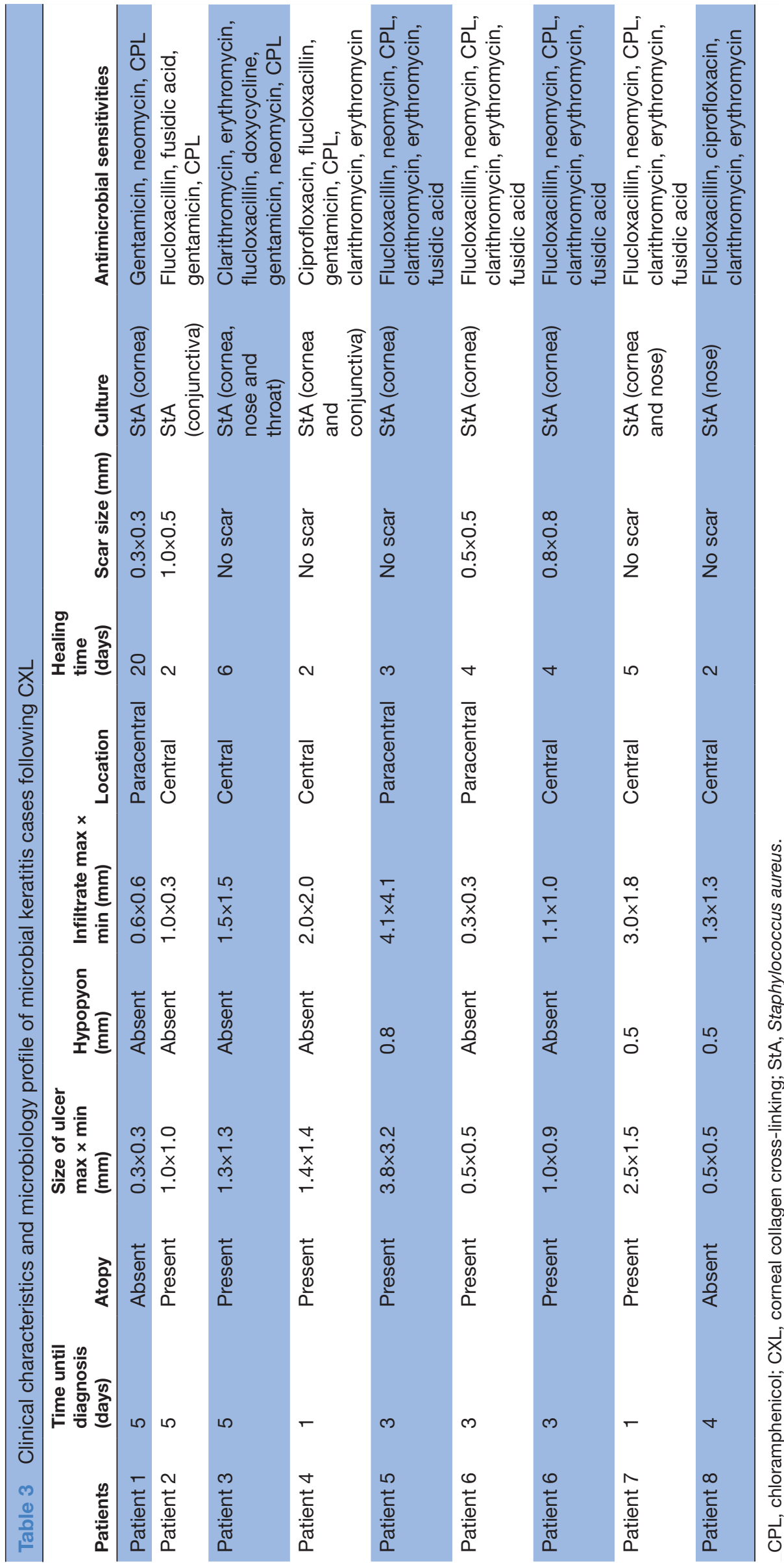



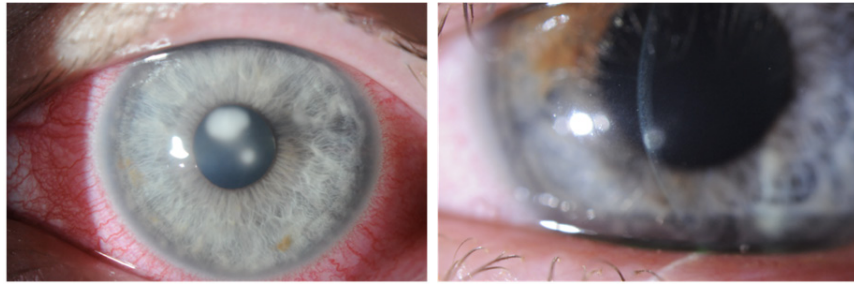

Figure 1 Corneal photographs of eyes with post-CXL keratitis. The left image shows a central corneal ulcer with infiltrate (case 3), while the right image shows a small early inferior paracentral corneal ulcer with respective infiltrate (case 6). CXL, corneal collagen cross-linking.

\section{DISCUSSION}

Although CXL is considered a relatively safe procedure, rare but sight-threatening complications may occur, including microbial keratitis, presumed sterile corneal infiltrates, corneal melting, corneal haze, scarring, herpes simplex virus keratitis and corneal endothelial damage. $^{10} 11$ 13-20 Until modifications can be made to improve the efficacy of transepithelial CXL, removal of the corneal epithelial barrier remains a risk factor for the development of microbial keratitis following standard CXL treatment.

Previous reports have identified poor postoperative treatment compliance and hygiene and the use of a BCL as possible risk factors for the development of microbial keratitis following CXL. ${ }^{8-111718}$ Maharana et $a l^{21}$ published a case series of seven microbial keratitis cases (incidence $1.3 \%$ ) after accelerated CXL with the use of a BCL, and concluded that younger age, atopic history, poor compliance and postoperative treatment may contribute to the development of keratitis. ${ }^{21}$ It is likely that changes in ocular flora and the use of topical steroids in patients with atopic or vernal conjunctivitis further increase the risk of postoperative keratitis. ${ }^{22}$ Shetty et $a l^{11}$ used a regimen in which a BCL was placed at the end of treatment, but steroids were deferred until epithelial healing had taken place. ${ }^{11}$ Despite the deferral of steroids, four patients still developed microbial keratitis, suggesting the BCL may

Table 4 Univariate analysis of the association between risk factors and development of microbial keratitis after CXL

\begin{tabular}{lcll}
\hline & OR & $\mathbf{9 5 \% ~ C l}$ & P value \\
\hline Age & 0.95 & 0.85 to 1.07 & 0.38 \\
Atopy & 3.68 & 0.76 to 17.79 & 0.07 \\
CTmin & 0.99 & 0.98 to 1.00 & 0.26 \\
Gender & 0.73 & 0.15 to 3.53 & 0.69 \\
Bilateral treatment & 0.3 & 0.04 to 2.45 & 0.26 \\
Postoperative BCL & 58.78 & 3.41 to 1012.89 & 0.005 \\
and steroids use & & & \\
Kmax & 1.09 & 0.97 to 1.23 & 0.15 \\
\hline
\end{tabular}

$\mathrm{BCL}$, bandage contact lens; CTmin, minimum preoperative corneal thickness; CXL, corneal collagen cross-linking; Kmax, maximum preoperative keratometric value. have been a greater risk factor for the development of microbial keratitis relative to the use of steroids.

The results of our study, which represents the largest up-to-date cohort on microbial keratitis following CXL, indicate that not using a BCL and withholding the use of topical steroids until the epithelium had healed after CXL (usually 2-5 days) were not associated with any cases of microbial keratitis. The limitations of the present study include its retrospective nature and the lack of a comparative measure of postoperative pain evaluation between the two study groups. Furthermore, due to the retrospective design of the study, it is not possible to discern whether the use of BCL, topical steroids or their combination has led to the increase in the incidence of microbial keratitis. Both of them have been identified so far in the literature as possible risk factors for the development of microbial keratitis after CXL. ${ }^{10} 11$ 17-21 In all of the cases described in the systematic review of Abbouda $e t a l,{ }^{10}$ the postoperative treatment included steroids, and in most of them also the use of a BCL. In two large retrospective cohorts using a BCL in all patients but deferring the use of steroids until complete epithelial healing, the incidence of MK after CXL varied between $0.17 \%(4 / 2350)$ and $1.3 \%$ (7/532). Further prospective randomised studies are needed in order to independently evaluate the risk and benefits of postoperative use of topical steroids and BCL.

It is of note that, in the original description, Wollensak et $a l^{\mathrm{l}}$ did not describe the use of either topical steroid nor of a BCL, but rather only topical antimicrobial therapy until the epithelium had healed. The results of this study would support that view. A variety of micro-organisms have been reported in cases of post-CXL microbial keratitis, with the spectrum including Escherichia coli, Aspergillus fumigatus, Pseudomonas aeruginosa, Streptococcus and Staphylococcus species, as well as Acanthamoeba spp.,${ }^{17-21}$ herpes simplex virus and fungi. ${ }^{10}$ It is of note, therefore, that in this study and in contrast to these reports, $S$. aureus was the only micro-organism isolated. In fact, $S$. aureus was cultured from corneal scrapes in seven out of nine cases and from contiguous sites in the remaining two cases. The clinical features in these two cases were consistent with microbial keratitis rather than sterile infiltrates.

Infections with $S$. aureus usually arise from endogenous sources and nasopharyngeal colonisation with $S$. aureus is a well-recognised risk factor. ${ }^{23-25}$ Approximately $20 \%$ of the healthy human population are persistently colonised with $S$. aureus and the same strain can persist over months or years. ${ }^{26}{ }^{27}$ A key feature of non-ocular $S$. aureus infection is its recurrence, which occurs in approximately $30 \%$ of all cases. ${ }^{28}{ }^{29}$ It is also of importance to note that $S$. aureus has been found to be associated with recurrent microbial keratitis significantly more frequently than with other bacteria. ${ }^{30}$ In addition to the nose, in some of our patients $S$. aureus was isolated from the ipsilateral conjunctiva. It is possible, therefore, that $S$. aureus may have persisted at or around the conjunctiva or lid margin in these patients as small colony variants, ${ }^{29}$ as these have 
been associated with persistent and recurrent infections at other sites. ${ }^{31} 32$

Persistent carriage of $S$. aureus in patients with keratoconus might therefore predispose these individuals to recurrent keratitis, especially in those with ocular surface disease such as atopic conjunctivitis, which was present in six of the eight patients who developed microbial keratitis. In patients with atopic or other ocular surface disease who are at an increased risk of microbial keratitis, collection of samples from the nose and conjunctiva prior to CXL followed by $S$. aureus decolonisation treatment prior to surgery is a consideration.

Interestingly, the combination of a large diameter BCL, antibiotics and steroids is often the treatment regimen of patients undergoing photorefractive keratectomy (PRK), and yet the keratitis rates in this group have been reported to be much lower, even less than $0.02 \% .^{33} 34$ As a matter of fact, Yuksel et $a l^{35}$ have reported comparable colonisation rates of BCLs after PRK and CXL, with coagulase-negative staphylococci being the most frequent micro-organism. ${ }^{35}$ Although CXL can also have a presumed antimicrobial effect on corneal stroma, the difference in microbial keratitis rates could be attributed to variations in aseptic surgical conditions and, moreover, to the increased incidence of atopy among patients with keratoconus in comparison with subjects undergoing refractive surgery.

In conclusion, our results would suggest that not using a BCL and delaying the introduction of topical steroids until epithelial healing may significantly reduce the risk of developing microbial keratitis and do not seem to increase the risk of persistent corneal haze. The role of preoperative sampling of the conjunctiva and nose and prophylactic antimicrobials requires further study.

Contributors Concept and design of the study: AT, VR, MB, CW, SA, SK. Data acquisition: AT, VR, RC, RV, TN. Data analysis/interpretation: AT, VR, RC, SA, SK. Drafting of the manuscript: AT, RC, VR, SK. Critical revision of the manuscript: AT, VR, RC, RV, MB, CW, TN, SA, SK. Statistical analysis: AT, SK. Admin, technical or material support: TN, VR, RV. Supervision: MB, CW, SK. Final approval: AT, VR, RC RV, MB, CW, TN, SA, SK.

Funding The authors have not declared a specific grant for this research from any funding agency in the public, commercial or not-for-profit sectors.

Competing interests None declared.

Patient consent Not required.

Ethics approval This retrospective cohort study was performed in adherence with the Declaration of Helsinki for research involving human subjects and after approval from the Institutional Review Board of The Royal Liverpool University Hospital.

Provenance and peer review Not commissioned; externally peer reviewed.

Open access This is an open access article distributed in accordance with the Creative Commons Attribution Non Commercial (CC BY-NC 4.0) license, which permits others to distribute, remix, adapt, build upon this work non-commercially, and license their derivative works on different terms, provided the original work is properly cited, appropriate credit is given, any changes made indicated, and the use is non-commercial. See: http://creativecommons.org/licenses/by-nc/4.0/.

\section{REFERENCES}

1. Wollensak G, Spoerl E, Seiler T. Riboflavin/ultraviolet-a-induced collagen crosslinking for the treatment of keratoconus. Am J Ophthalmology 2003;135:620-7.
2. Li J, Ji P, Lin X. Efficacy of corneal collagen cross-linking for treatment of keratoconus: a meta-analysis of randomized controlled trials. PLoS One 2015;10:e0127079.

3. O'Brart DPS, Chan E, Samaras $\mathrm{K}$, et al. A randomised, prospective study to investigate the efficacy of riboflavin/ultraviolet A (370 $\mathrm{nm}$ ) corneal collagen cross-linkage to halt the progression of keratoconus. Br J Ophthalmol 2011;95:1519-24.

4. Vinciguerra R, Romano MR, Camesasca FI, et al. Corneal crosslinking as a treatment for keratoconus: four-year morphologic and clinical outcomes with respect to patient age. Ophthalmology 2013;120:908-16.

5. Raiskup F, Theuring A, Pillunat LE, et al. Corneal collagen crosslinking with riboflavin and ultraviolet-A light in progressive keratoconus: Ten-year results. J Cataract Refract Surg 2015;41:41-6.

6. Caporossi A, Mazzotta C, Paradiso AL, et al. Transepithelial corneal collagen crosslinking for progressive keratoconus: 24-month clinical results. J Cataract Refract Surg 2013;39:1157-63.

7. Soeters N, Wisse RPL, Godefrooij DA, et al. Transepithelial versus epithelium-off corneal cross-linking for the treatment of progressive keratoconus: a randomized controlled trial. Am J Ophthalmol 2015;159:821-8.

8. Koller T, Mrochen M, Seiler T. Complication and failure rates after corneal crosslinking. J Cataract Refract Surg 2009;35:1358-62.

9. Spoerl E, Mrochen M, Sliney D, et al. Safety of UVA-riboflavin crosslinking of the cornea. Cornea 2007;26:385-9.

10. Abbouda A, Abicca I, Alió JL. Infectious keratitis following corneal crosslinking: a systematic review of reported cases: management, visual outcome, and treatment proposed. Semin Ophthalmol 2016;31:485-91.

11. Shetty R, Kaweri L, Nuijts RMMA, et al. Profile of microbial keratitis after corneal collagen cross-linking. Biomed Res Int 2014;2014:1-7. Article ID 340509,

12. Vinciguerra $R$, Romano $V$, Arbabi EM, et al. In vivo early corneal biomechanical changes after corneal cross-linking in patients with progressive keratoconus. J Refract Surg 2017;33:840-6.

13. Angunawela RI, Arnalich-Montiel F, Allan BDS. Peripheral sterile corneal infiltrates and melting after collagen crosslinking for keratoconus. J Cataract Refract Surg 2009;35:606-7.

14. Raiskup F, Hoyer A, Spoerl E. Permanent corneal haze after riboflavin-UVA-induced cross-linking in keratoconus. J Cataract Refract Surg 2009;25:S824-S828.

15. Kymionis GD, Portaliou DM, Bouzoukis DI, et al. Herpetic keratitis with iritis after corneal crosslinking with riboflavin and ultraviolet $A$ for keratoconus. J Cataract Refract Surg 2007;33:1982-4.

16. Wollensak G, Spoerl E, Wilsch M, et al. Endothelial cell damage after riboflavin-ultraviolet-A treatment in the rabbit. $J$ Cataract Refract Surg 2003;29:1786-90.

17. Pollhammer $M$, Cursiefen $C$. Bacterial keratitis early after corneal crosslinking with riboflavin and ultraviolet-A. J Cataract Refract Surg 2009;35:588-9.

18. Sharma N, Maharana P, Singh G, et al. Pseudomonas keratitis after collagen crosslinking for keratoconus: case report and review of literature. J Cataract Refract Surg 2010;36:517-20.

19. Zamora KV, Males JJ. Polymicrobial keratitis after a collagen crosslinking procedure with postoperative use of a contact lens: a case report. Cornea 2009;28:474-6.

20. Rama P, Di Matteo F, Matuska S, et al. Acanthamoeba keratitis with perforation after corneal crosslinking and bandage contact lens use. $J$ Cataract Refract Surg 2009;35:788-91.

21. Maharana PK, Sahay P, Sujeeth M, et al. Microbial keratitis after accelerated corneal collagen cross-linking in keratoconus. Cornea 2018;37:162-7

22. Ermis SS, Aktepe OC, Inan UU, et al. Effect of topical dexamethasone and ciprofloxacin on bacterial flora of healthy conjunctiva. Eye 2004;18:249-52.

23. Lakhundi S, Siddiqui R, Khan NA. Pathogenesis of microbial keratitis. Microb Pathog 2017;104:97-109.

24. von Eiff C, Becker K, Machka K, et al. Nasal carriage as a source of Staphylococcus aureus bacteremia. Study Group. N Engl J Med 2001;344:11-16.

25. Wertheim HFL, Vos MC, Ott A, et al. Risk and outcome of nosocomial Staphylococcus aureus bacteraemia in nasal carriers versus non-carriers. The Lancet 2004;364:703-5.

26. Weidenmaier C, Goerke C, Wolz C. Staphylococcus aureus determinants for nasal colonization. Trends in Microbiology 2012;20:243-50.

27. van Belkum A, Verkaik NJ, de Vogel CP, et al. Reclassification of Staphylococcus aureus nasal carriage types. J Infect Dis 2009:199:1820-6. 
28. Sakwinska O, Blanc DS, Lazor-Blanchet C, et al. Ecological temporal stability of Staphylococcus aureus nasal carriage. J Clin Microbiol 2010;48:2724-8.

29. Proctor RA, van Langevelde P, Kristjansson M, et al. Persistent and relapsing infections associated with small-colony variants of Staphylococcus aureus. Clinical Infectious Diseases 1995;20:95-102.

30. Kaye R, Kaye A, Sueke H, et al. Recurrent bacterial keratitis. Invest Ophthalmol Vis Sci 2013;54:4136-9.

31. Kim HK, Thammavongsa V, Schneewind O, et al. Recurrent infections and immune evasion strategies of Staphylococcus aureus. Current Opinion in Microbiology 2012;15:92-9.
32. Proctor RA, von Eiff C, Kahl BC, et al. Small colony variants: a pathogenic form of bacteria that facilitates persistent and recurrent infections. Nat Rev Microbiol 2006;4:295-305.

33. Wroblewski KJ, Pasternak JF, Bower KS, et al. Infectious keratitis after photorefractive keratectomy in the United States Army and navy. Ophthalmology 2006;113:520-5.

34. Schallhorn JM, Schallhorn SC, Hettinger K, et al. Infectious keratitis after laser vision correction: incidence and risk factors. J Cataract Refract Surg 2017;43:473-9.

35. Yuksel E, Yalcin NG, Kilic G, et al. Microbiologic examination of bandage contact lenses used after corneal collagen cross-linking treatment. Ocul Immunol Inflamm 2016;24:217-2. 